\title{
PRINCIPAL CONGRUENCES ON DISTRIBUTIVE DOUBLE $p$-ALGEBRAS
}

\author{
by JÚlia VAZ DE CARVALHO*
}

(Received 29th September 1994)

\begin{abstract}
We use Priestley's duality to characterize, via their dual space, the distributive double p-algebras on which all congruences are principal.
\end{abstract}

1991 Mathematics subject classification: 06D15.

\section{Introduction}

An algebra $L$ is said to have property $P C$ if all its congruences are principal. This notion was introduced by Blyth and Varlet in [3]. There the authors characterize the distributive lattices and the Stone, the de Morgan and the Heyting algebras that have $P C$.

In [2], Beazer characterizes the quasi-modular $p$-algebras that have $P C$ and solves the same problem for some special classes of distributive double $p$-algebras, using purely algebraic tools.

In this paper we use Priestley's duality to characterize, via their dual space, all the distributive double $p$-algebras that have $P C$. As a consequence we obtain not only the characterization for double Stone algebras established by the author in [9] and by Beazer in [2], but also the characterizations stated in [2] for some special classes of distributive double $p$-algebras.

In order to obtain such results, we start by determining the subsets of the dual space of a distributive double $p$-algebra that represent principal congruences and also "translate" some properties on the dual space into properties of the algebra and vice versa.

\section{Preliminaries}

A distributive $p$-algebra is an algebra $A=(A ; \wedge, \vee, *, 0,1)$ of type $(2,2,1,0,0)$ such that $(A ; \wedge, \vee, 0,1)$ is a bounded distributive lattice and $*$ is a unary operation that satisfies

*This work was done within the activities of Centro de Álgebra da Universidade de Lisboa. 


$$
y \wedge x=0 \Leftrightarrow y \leqq x^{*},
$$

i.e., $x^{*}$ is the pseudocomplement of $x$.

A distributive double $p$-algebra is an algebra $A=(A ; \wedge, \vee, *,+, 0,1)$ of type $(2,2,1,1,0,0)$ such that $A=(A ; \wedge, \vee, *, 0,1)$ is a distributive $p$-algebra and + is a unary operation that satisfies

$$
y \vee x=1 \Leftrightarrow x^{+} \leqq y
$$

i.e., $x^{+}$is the dual psudocomplement of $x$.

We denote the variety of distributive double $p$-algebras by $\mathbf{B}_{\omega}^{\omega}$.

Let $L$ be a distributive (double) $p$-algebra. We say that $L$ is a (double) Stone algebra if $x^{*} \vee x^{* *}=1\left(x^{*} \vee x^{* *}=1\right.$ and $\left.x^{+} \wedge x^{++}=0\right)$, for every $x \in L$.

We start by giving a brief outline of Priestley's duality as it applies to distributive double $p$-algebras. For more details see $[4,5,6,7]$.

First, let $P$ be a partially ordered set and $Q \subseteq P$. We define $\uparrow Q=\{x \in P \mid(\exists y \in Q) x \geqq y\}$ and $\downarrow Q=\{x \in P \mid(\exists y \in Q) x \leqq y\}$. When $Q=\{z\}$, we simply write $\uparrow z$ and $\downarrow z$ for $\uparrow\{z\}$ and $\downarrow\{z\}$, respectively. The subset $Q$ is said to be an up set if $Q=\uparrow Q$, a down set if $Q=\downarrow Q$ and an up-down set if $Q=\uparrow Q=\downarrow Q$. We denote by $\operatorname{Min} P$ and Max $P$ the sets of minimal and of maximal elements of $P$, respectively. We say that $P$ has length less than or equal to $k \in N_{0}$, and write $l(P) \leqq k$, if every chain in $P$ has at most $k+1$ elements. Let $X=(X ; \tau, \leqq)$ be an ordered topological space, $X$ is called a Priestley space if it is compact and totally order disconnected, that is, if $x, y \in X$ and $x \neq y$, there exists a clopen down set $U$ such that $y \in U$ and $x \notin U$ (thus $X$ is Hausdorff). If $X$ is a Priestley space, then, for every $x \in X$, there exist $y \in \operatorname{Min} X$ and $z \in \operatorname{Max} X$ such that $y \leqq x \leqq z$ and, for every closed set $Q$, the subsets $\uparrow Q$ and $\downarrow Q$ are closed.

Now, we say that a Priestley space $X$ is a double p-space if $\uparrow U$ and $\downarrow V$ are open (and therefore clopen), whenever $U$ is a clopen down subset of $X$ and $V$ is a clopen up subset of $X$. Let $X_{1}$ and $X_{2}$ be double $p$-spaces. A map $f: X_{1} \rightarrow X_{2}$ is said to be a double p-morphism if it is continuous, order-preserving and $f\left(\operatorname{Min} X_{1} \cap \downarrow x\right)=\operatorname{Min} X_{2} \cap \downarrow f(x)$ and $f\left(\operatorname{Max} X_{1} \cap \uparrow x\right)=\operatorname{Max} X_{2} \cap \uparrow f(x)$, for every $x \in X_{1}$. Next, notice that the category of distributive double $p$-algebras together with homomorphisms is dually equivalent to the category of double $p$-spaces together with double $p$-morphisms. Given a double $p$ space $X$, its dual algebra $\mathcal{O}(X)$ is the distributive double $p$-algebra whose elements are the clopen down subsets of $X$ and whose operations are the intersection, union, $\emptyset, X$ and $*$ and + defined as follows: $U^{*}=X-\uparrow U$ and $U^{+}=\downarrow(X-U)$, for every clopen down set $U$. Given a distributive double $p$-algebra $L$, its dual space is $(X ; \tau, \leqq)$ where $X$ is the set of prime ideals of $L$, the topology $\tau$ has as a sub-basis $\left\{X_{a} \mid a \in L\right\} \cup\left\{X-X_{a} \mid\right.$ $a \in L\}$ (for each $a \in L, X_{a}=\{I \in X \mid a \notin I\}$ ) and the order is the inclusion. If $X$ is a double $p$-space, then a subset $Q$ of $X$ is called a double $p$-subset if $\uparrow(Q \cap \operatorname{Min} X) \subseteq Q$ and $\downarrow(Q \cap \operatorname{Max} X) \subseteq Q$. There is an isomorphism $\psi$ between the congruence lattice of $L \in \mathbf{B}_{\omega}^{\omega}$ and the lattice of open double $p$-subsets of its dual space $X$ which assigns to each congruence $\theta$ the open double $p$-subset $\bigcup_{(a, b) \in \theta}\left(X_{a}-X_{b}\right)$. If $Q$ is an open double $p$-subset of $X$, then $(a, b) \in \psi^{-1}(Q)$ if and only if $X_{a}-Q=X_{b}-Q$. We ought to observe 
that in [4] and [6] the authors represent the congruences by closed sets whereas we do it by open sets; we just take the complements.

Let $X$ be a double $p$-space. In [8, Lemma 1, Corollary 6], Priestley showed that $\operatorname{Min} X$ is closed in $X$ and if $C$ is a clopen subset of $X$, then $\uparrow(C \cap \operatorname{Min} X)$ is clopen. Similarly, we can prove that $\operatorname{Max} X$ is closed in $X$ and $\downarrow(C \cap \operatorname{Max} X)$ is clopen, whenever $C$ is clopen. We denote by $\operatorname{Mid} X$ the open double $p$-subset $X-(\operatorname{Min} X \cup \operatorname{Max} X)$. This set plays an important role since it represents the determination congruence of $\mathcal{O}(X)$, i.e., for all $U, V \in \mathcal{O}(X)$,

$$
U-\operatorname{Mid} X=V-\operatorname{Mid} X \Leftrightarrow U^{*}=V^{*} \text { and } U^{+}=V^{+} .
$$

There is an isomorphism between the lattice of filters of $L \in \mathbf{B}_{\omega}^{\omega}$ and the lattice of open up subsets of its dual space $X$ which assigns to each filter $F$ of $L$ the open up set $\Gamma_{F}=\bigcup_{a \in F}\left(X-X_{a}\right)$. By [6, Theorem 2.3.5], the filter $F$ is normal (i.e., $a^{+*} \in F$, whenever $a \in F)$ if and only if $\Gamma_{F}$ is a down set.

By [7, Proposition 3], if $X$ is the dual space of a double Stone algebra, then, for every $x \in X$, there is a unique $m(x) \in \operatorname{Min} X$ such that $m(x) \leqq x$; similarly, we prove that there is a unique $M(x) \in \operatorname{Max} X$ such that $x \leqq M(x)$. Notice that the partial order we defined on $X$ is the reverse of that used in [7].

If $L$ is a finite distributive double $p$-algebra, we may consider its dual space to be the pair $(J(L)$; $\leqq$, where $J(L)$ is the set of nonzero join irreducible elements of $L$ and $\leqq$ is the partial order induced by the lattice order. Here we drop the topology as it is the discrete one.

\section{Double p-algebras}

Let $X$ be a double $p$-space and $C \subseteq X$. For $n \in N_{0}$, we define $B_{n}(C), B_{n}^{\prime}(C)$ and $(\uparrow \downarrow)^{n}(C)$ as follows:

$$
\begin{gathered}
B_{0}(C)=B_{0}^{\prime}(C)=C,(\uparrow \downarrow)^{0}(C)=C, \\
B_{n+1}(C)=\downarrow\left(B_{n}^{\prime}(C) \cap \operatorname{Max} X\right), B_{n+1}^{\prime}(C)=\uparrow\left(B_{n}(C) \cap \operatorname{Min} X\right) \text { and }(\uparrow \downarrow)^{n+1}(C)=\uparrow \downarrow\left((\uparrow \downarrow)^{n}(C)\right) .
\end{gathered}
$$

We define $(\downarrow \uparrow)^{n}(C)$ similarly. For every $n \in N$, it is obvious that $B_{n}(C)$ is a down set and $B_{n}^{\prime}(C)$ is an up set.

Lemma 3.1. Let $X$ be a double $p$-space and $C \subseteq X$.

(i) If $C$ is a down set, then $\uparrow(C \cap \operatorname{Min} X)=\uparrow C$;

(iii) If $C$ is an up set, then $\downarrow(C \cap \operatorname{Max} X)=\downarrow C$;

(iii) For every $n \in N, B_{n}^{\prime}(C) \subseteq B_{n+1}(C)$ and $B_{n}(C) \subseteq B_{n+1}^{\prime}(C)$;

(iv) For every $n \geqq 3, B_{n}(C)=\downarrow \uparrow B_{n-2}(C)$;

(v) If $C$ is an up set, then $\bigcup_{n \in N_{0}} B_{n}(C)=\bigcup_{n \in N_{0}}(\uparrow \downarrow)^{n}(C)$; 
(vi) If $C$ is clopen, then, for every $n \in N_{0}, B_{n}(C)$ is clopen and $\bigcup_{n \in N_{0}} B_{n}(C)$ is open;

(vii) $Q=\bigcup_{n \in N_{0}} B_{n}(C)$ is a double p-subset of $X$;

(viii) If $X$ is the dual space of a double Stone algebra, then $\bigcup_{n \in N_{0}} B_{n}(C)=\bigcup_{n=0}^{2} B_{n}(C)$.

Proof. (i) Let $y \in \uparrow C$. There exist $c \in C$ and $m \in \operatorname{Min} X$ such that $m \leqq c \leqq y$. Since $C$ is a down set, the element $m \in C \cap \operatorname{Min} X$. Therefore, $y \in \uparrow(C \cap \operatorname{Min} X)$.

The proof of (ii) is similar.

(iii) Let $n \in N$. By the definitions of the $B s, B^{\prime} s$ and (i) and (ii),

$$
\begin{aligned}
& B_{n+1}(C)=\downarrow\left(B_{n}^{\prime}(C) \cap \operatorname{Max} X\right)=\downarrow B_{n}^{\prime}(C) \supseteq B_{n}^{\prime}(C), \\
& B_{n+1}^{\prime}(C)=\uparrow\left(B_{n}(C) \cap \operatorname{Min} X\right)=\uparrow B_{n}(C) \supseteq B_{n}(C) .
\end{aligned}
$$

(iv) Let $n \geqq 3$. By the definitions of the $B s, B^{\prime} s$ and (i) and (ii), we get

$$
B_{n}(C)=\downarrow\left(B_{n-1}^{\prime}(C) \cap \operatorname{Max} X\right)=\downarrow B_{n-1}^{\prime}(C)=\downarrow \uparrow\left(B_{n-2}(C) \cap \operatorname{Min} X\right)=\downarrow \uparrow B_{n-2}(C) \text {. }
$$

(v) Let $C$ be an up set. First we prove, inductively, that, for every $n \in N$, we have $B_{2 n}(C) \subseteq B_{2 n-1}(C)$. Since $C$ is an up set, then, by the definition of the $B s, B^{\prime} s$ and (ii),

$B_{2}(C)=\downarrow\left(B_{1}^{\prime}(C) \cap \operatorname{Max} X\right)=\downarrow B_{1}^{\prime}(C)=\downarrow \uparrow(C \cap \operatorname{Min} X) \subseteq \downarrow \uparrow C=\downarrow C=\downarrow(C \cap \operatorname{Max} X)=B_{1}(C)$.

Suppose

$B_{2 n}(C) \subseteq B_{2 n-1}(C)$. By (iv), we have $B_{2 n+2}(C)=\downarrow \uparrow B_{2 n}(C) \subseteq \downarrow \uparrow B_{2 n-1}(C)=B_{2 n+1}(C)$.

Now, applying (ii) and (iv),

$$
\bigcup_{n \in N_{0}} B_{n}(C)=\bigcup_{n \in N_{0}} B_{2 n+1}(C)=\bigcup_{n \in N_{0}}(\downarrow \uparrow)^{n}(\downarrow C)=\bigcup_{n \in N_{0}}(\uparrow \downarrow)^{n}(C) \text {. }
$$

(vi) By hypothesis, $B_{0}(C)=B_{0}^{\prime}(C)=C$ is clopen. Now, it is clear, from the observations in Section 2, that if $B_{n}(C)$ and $B_{n}^{\prime}(C)$ are clopen, so are $B_{n+1}(C)$ and $B_{n+1}^{\prime}(C)$. Therefore, for every $n \in N_{0}$, the set $B_{n}$ is clopen and the set $\bigcup_{n \in N_{0}} B_{n}(C)$ is open.

(vii) The set

$$
\uparrow(Q \cap \operatorname{Min} X)=\uparrow\left(\left(\bigcup_{n \in N_{0}} B_{n}(C)\right) \cap \operatorname{Min} X\right)=\bigcup_{n \in N_{0}} \uparrow\left(B_{n}(C) \cap \operatorname{Min} X\right)=\bigcup_{n \in N} B_{n}^{\prime}(C)
$$

is contained in $Q$, by (iii). And

$$
\begin{aligned}
\downarrow(Q \cap \operatorname{Max} X) & =\downarrow\left(\left(\bigcup_{n \in N_{0}} B_{n}(C)\right) \cap \operatorname{Max} X\right)=\bigcup_{n \in N_{0}} \downarrow\left(B_{n}(C) \cap \operatorname{Max} X\right) \\
& \subseteq \downarrow\left(B_{0}(C) \cap \operatorname{Max} X\right) \cup \bigcup_{n \in N} \downarrow B_{n}(C) \\
& =\downarrow\left(B_{0}^{\prime}(C) \cap \operatorname{Max} X\right) \cup \bigcup_{n \in N} B_{n}(C) \\
& =\bigcup_{n \in N} B_{n}(C) \subseteq Q .
\end{aligned}
$$


(viii) It is sufficient to show that $B_{n}(C) \subseteq B_{n-2}(C)$, for $n \geqq 3$. Let $n \geqq 3$ and $y \in B_{n}(C)$. By the definitions of $B_{n}(C), B_{n-1}^{\prime}(C)$ and $B_{n-2}(C)$, there are elements $x \in B_{n-1}^{\prime}(C) \cap \operatorname{Max} X$, $z \in B_{n-2}(C) \cap \operatorname{Min} X$ and $t \in B_{n-3}^{\prime}(C) \cap \operatorname{Max} X$ such that $z \leqq t, z \leqq x$ and $y \leqq x$. But $t, x \in \operatorname{Max} X$, and so $t=x$ and $y \in \downarrow\left(B_{n-3}^{\prime}(C) \cap \operatorname{Max} X\right)=B_{n-2}(C)$.

Proposition 3.2. Let $L \in \mathbf{B}_{\omega}^{\omega}$ and $a, b \in L, a \leqq b$.

(i) The congruence $\theta(a, b)$ is represented by $Q=\bigcup_{n \in N_{0}} B_{n}\left(X_{b}-X_{a}\right)$;

(ii) If $L$ is a double Stone algebra, then $\theta(a, b)$ is represented by the clopen set $Q=\bigcup_{n=0}^{2} B_{n}\left(X_{b}-X_{a}\right)$.

Proof. (i) First notice that the open double $p$-subset of $X$ that represents $\theta(a, b)$ must contain

$$
Q=\bigcup_{n \in N_{0}} B_{n}\left(X_{b}-X_{a}\right)
$$

Hence, it is sufficient to show that $Q$ is an open double $p$-subset of $X$ but this follows from Lemma 3.1 (vi) and (vii).

(ii) This is an immediate consequence of (i) and Lemma 3.1 (viii), (vi).

Proposition 3.3. Let $L \in \mathbf{B}_{\omega}^{\omega}$ and $X$ be its dual space.

(i) The subset $Q$ of $X$ represents a principal congruence if and only if there is a clopen convex set $C$ such that $Q=\bigcup_{n \in N_{0}} B_{n}(C)$;

(ii) If $L$ is a double Stone algebra, then the subset $Q$ of $X$ represents a principal congruence if and only if there is a clopen convex set $C$ such that $Q=\bigcup_{n=0}^{2} B_{n}(C)$.

Proof. (i) Suppose that $Q$ represents a principal congruence $\theta(a, b)$. We may suppose that $a \leqq b$. By Proposition 3.2, we have $Q=\bigcup_{n \in N_{0}} B_{n}\left(X_{b}-X_{a}\right)$ and $X_{b}-X_{a}$ is clopen and convex.

Conversely, let $Q=\bigcup_{n \in N_{0}} B_{n}(C)$, for some clopen convex set $C$. Then $Q$ is an open double p-subset, by Lemma 3.1 (vi) and (vii). Now, the result follows as in Lemma 3 of [1].

(ii) This follows from (i) and Lemma 3.1 (viii).

Proposition 3.4. If $L \in \mathrm{B}_{\omega}^{\omega}$ and $L$ has $P C$, then $D$ is an open up-down subset of its dual space $X$ if and only if there is a clopen up set $A$ such that $D=\bigcup_{n \in N_{0}}(\uparrow \downarrow)^{n}(A)$.

Proof. Let $D$ be an open up-down set, then $D$ represents a congruence on $L$ which must be principal. By Proposition 3.3 (i), there is a clopen convex set $C$ such that $D=\bigcup_{n \in N_{0}} B_{n}(C)$. However, $D$ is an open up-down set, and so it also represents a filter $F$ of $L$, that is, $D=\bigcup_{a \in F}\left(X-X_{a}\right)$. The clopen $C$ is contained in $\bigcup_{a \in F}\left(X-X_{a}\right)$. Since $X$ is compact, there exist $t \in N_{0}$ and $a_{1}, \ldots, a_{t} \in F$ such that $C$ is contained in 
$\bigcup_{1 \leqq i \leqq t}\left(X-X_{a_{i}}\right)=X-X_{a_{1}} \wedge \ldots \wedge a_{t}$. Let $b=a_{1} \wedge \ldots \wedge a_{t}$ and $A=X-X_{b}$. Then the element $b \in F$ and $D$ is up and down, hence $\bigcup_{n \in N_{0}}(\uparrow \downarrow)^{n}(A) \subseteq D$. By applying Lemma 3.1 (v) to the up set $A$, we obtain $D=\bigcup_{n \in N_{0}} B_{n}(C) \subseteq \bigcup_{n \in N_{0}} B_{n}(A)=\bigcup_{n \in N_{0}}(\uparrow \downarrow)^{n}(A)$.

Now, let $D=\bigcup_{n \in N_{0}}(\uparrow \downarrow)^{n}(A)$, where $A$ is a clopen up set. Clearly $D$ is up and down, hence, by Lemma $3.1(\mathrm{v})$ and (vi), $D$ is open.

Proposition 3.5. Let $L \in \mathbf{B}_{\omega}^{\omega}$ and $X$ be its dual space. If $L$ has $P C$, then every subset of Mid $X$ is clopen and convex.

Proof. First we show that if $Q \subseteq \operatorname{Mid} X$ and $Q$ is open in $X$, then $Q$ is clopen and convex. Let $Q \subseteq \operatorname{Mid} X$ and $Q$ be open. Then $Q$ represents a congruence on $L$ which must be principal. By Proposition 3.3 (i), there is a clopen convex set $C$ such that $Q=\bigcup_{n \in N_{0}} B_{n}(C)$. But, for every $n \in N$, we have $B_{n}(C)=\emptyset$. Therefore $Q=C$ and $Q$ is clopen and convex.

To complete the proof it remains to show that every subset of Mid $X$ is open. Let $x \in \operatorname{Mid} X$, the set $\operatorname{Mid} X-\{x\}$ is open and so it is clopen. Thus $\{x\}=\operatorname{Mid} X-(\operatorname{Mid} X-\{x\})$ is open.

Corollary 3.6. Let $L \in \mathbf{B}_{\omega}^{\omega}$ and $X$ be its dual space. If $L$ has $P C$, then $\operatorname{Mid} X$ is finite.

Proof. By Proposition 3.5, the set $\operatorname{Mid} X$ is closed and every subset of $\operatorname{Mid} X$ is clopen in $X$ and so clopen in Mid $X$. Thus Mid $X$ is a compact Hausdorff space whose subsets are clopen. Therefore, by [5, Lemma 10.9A], Mid $X$ is finite.

Corollary 3.7. Let $L \in \mathbf{B}_{\omega}^{\omega}$ and $X$ be its dual space. If $L$ has $P C$, then $l(X) \leqq 3$.

Proof. Suppose that $l(X)>3$. Then, there are elements $a_{1}, a_{2}, a_{3}, a_{4}, a_{5} \in X$ such that $a_{1}<a_{2}<a_{3}<a_{4}<a_{5}$. Hence, the set $\left\{a_{2}, a_{4}\right\}$ is contained in Mid $X$ and is not convex. This contradicts Proposition 3.5.

Lemma 3.8. Let $X$ be a double p-space such that $l(X) \leqq 3$ and $\operatorname{Mid} X$ is finite. Let $A$ and $T$ be subsets of $X$ and $\operatorname{Mid} X$, respectively, and $T_{1}=\{x \in T \mid(\exists y \notin A \cup T)(\exists z \in A) x<y<z\}$. If $A$ is a clopen up set and $T$ is open, then $A \cup\left(T-T_{1}\right)$ is clopen and convex.

Proof. The sets $T, T_{1}$ and $T-T_{1}$ are closed, since $\operatorname{Mid} X$ is finite and $T, T_{1}$ and $T-T_{1}$ are subsets of Mid $X$. However $T$ is open and so $T-T_{1}$ is also open. Therefore, $A \cup\left(T-T_{1}\right)$ is clopen.

To show that $A \cup\left(T-T_{1}\right)$ is convex, let $u, v \in A \cup\left(T-T_{1}\right)$ and $x \in X$ be such that $u<x<v$. If $u \in A$, then $x \in A$, since $A$ is an up set. Suppose that $u \in T-T_{1}$. Now, as $l(X) \leqq 3$ and $T-T_{1} \subseteq \operatorname{Mid} X$, the element $v$ must belong to $A$. If $x \notin A \cup T$, then $u \in T_{1}$, a contradiction. If $x \in T_{1}$, then there are elements $y \notin A \cup T$ and $z \in A$ such that $u<x<y<z$, contradicting the fact that $u \in T-T_{1}$. Thus $x \in A \cup\left(T-T_{1}\right)$ and so $A \cup\left(T-T_{1}\right)$ is convex. 
Theorem 3.9. Let $L \in \mathbf{B}_{\omega}^{\omega}$ and $X$ be its dual space. Then $L$ has $P C$ if and only if $X$ satisfies the following conditions

(i) $\mathrm{Mid} X$ is finite;

(ii) $l(X) \leqq 3$;

(iii) The open up-down subsets of $X$ are the sets of the form $\bigcup_{n \in N_{0}}(\uparrow \downarrow)^{n}(A)$, where $A$ is a clopen up set.

Proof. Suppose that $L$ has PC. By Proposition 3.4 and Corollaries 3.6 and 3.7, the conditions (i), (ii) and (iii) are satisfied.

Conversely, suppose that $X$ satisfies (i), (ii) and (iii) and let $Q \subseteq X$ be an open double $p$-subset of $X$. By [6, Theorems 2.3.5, 2.3.6, 2.3.7], we have $Q=Q_{1} \cup(Q \cap \operatorname{Mid} X)$, where $Q_{1}$ is an open up-down set. Hence, there is a clopen up set $A$ such that $Q_{1}=$ $\bigcup_{n \in N_{0}}(\uparrow \downarrow)^{n}(A)$. By Lemma $3.1(\mathrm{v})$, we obtain $Q_{1}=\bigcup_{n \in N_{0}} B_{n}(A)$. Next, notice that the set $T=Q \cap$ Mid $X$ is open and so, by Lemma 3.8, the set $A \cup\left(T-T_{1}\right)$ is clopen and convex. If $x \in T_{1}$, then $x \in \downarrow A=B_{1}(A)$, by the definition of $T_{1}$ and Lemma 3.1 (ii). Therefore

$$
Q=\left(\bigcup_{n \in N_{0}} B_{n}(A)\right) \cup T=\left(\bigcup_{n \in N_{0}} B_{n}(A)\right) \cup\left(T-T_{1}\right)=\bigcup_{n \in N_{0}} B_{n}\left(A \cup\left(T-T_{1}\right)\right)
$$

and, by Proposition 3.3 (i), it follows that $Q$ represents a principal congruence. Hence $L$ has $P C$.

Corollary 3.10. Let $L$ be a double Stone algebra and $X$ be its dual space. Then $L$ has $P C$ if and only if $X$ is finite and $l(X) \leqq 3$ (if and only if $L$ is finite and $l(J(L)) \leqq 3$ ).

Proof. Suppose that $L$ has $P C$. By Theorem 3.9, we know that $\operatorname{Mid} X$ is finite and $l(X) \leqq 3$. Let $x \in \operatorname{Min} X$. The set $\uparrow x$ is closed. Consider the open set $X-\uparrow x$. It is easy to prove that $X-\uparrow x$ is a double $p$-subset. Thus $X-\uparrow x$ represents a congruence that must be principal. By Proposition 3.2 (ii), the set $X-\uparrow x$ is clopen and so $\uparrow x$ is open and $\{x\}=\uparrow x \cap \operatorname{Min} X$ is open in Min $X$. Now, every subset of the compact Hausdorff space Min $X$ is open and, by [5, Lemma 10.9A], the set $\operatorname{Min} X$ is finite. In a similar way we prove that $\operatorname{Max} X$ is also finite. Therefore, $X=\operatorname{Min} X \cup \operatorname{Max} X \cup \operatorname{Mid} X$ is finite.

The converse is immediate, by Theorem 3.9.

The following examples prove that conditions (i), (ii) and (iii) of Theorem 3.9 are independent.

1. Consider $L$ the 6-element chain with $1^{+}=0$ and $x^{+}=1$, for every $x \neq 1 ; 0^{*}=1$ and $x^{*}=0$, for every $x \neq 0$. Its dual space $X$ is the five element chain: $x_{0}<x_{1}<x_{2}<x_{3}<x_{4}$. Now, $X$ satisfies conditions (i) and (iii), but it does not satisfy (ii). Notice that $L$ does not have $P C:\left\{x_{1}, x_{3}\right\}$ represents a congruence which is not principal.

2. Let $\{0\}$ and $\{1\}$ be one point double $p$-spaces and $N \infty=\left\{x_{n} \mid n \in N \cup\{\infty\}\right\}$ be the 
double $p$-space defined by the partial order given by $x_{i} \| x_{j}$, for all $i, j \in N \cup\{\infty\}, i \neq j$, and the one-point compactification of a countable discrete space, [5, Example 10.11]. Consider $X=\{0\} \oplus N \infty \oplus\{1\}$, where $\oplus$ denotes the usual linear sum and which is endowed with the disjoint union topology [5, Exercise 10.4]. Now, $X$ satisfies (ii) and (iii), but it does not satisfy (i). The dual double $p$-algebra of $X$ does not have $P C$ : $N \infty-\left\{x_{\infty}\right\}$ represents a congruence which is not principal.

3. Let $N \infty$ be the double $p$-space defined in the previous example. Obviously, $N \infty$ satisfies (i) and (ii), but it does not satisfy (iii): $\left\{x_{n} \mid n \in N\right\}$ is an open up-down subset of $N \infty$ and there is no clopen up set $A$ of $N \infty$ such that $\left\{x_{n} \mid n \in N\right\}=\bigcup_{n \in N}(\uparrow \downarrow)^{n}(A)$. The double $p$-algebra $L$, dual of $N \infty$, does not have $P C$ : the underlying lattice is a Boolean lattice and every lattice congruence is a congruence of $L$, thus by $[3$, Theorem 1] $L$ does not have $P C$. The set $\left\{x_{n} \mid n \in N\right\}$ represents a congruence which is not principal.

In [2], Beazer characterizes some classes of distributive double $p$-algebras that have $P C$. In order to show that his characterizations follow from our Theorem 3.9, we must translate some properties of distributive double $p$-algebras into properties of its dual space and vice-versa.

Let $L \in \mathbf{B}_{\omega}^{\omega}$. The dually dense set of $L$ is $D^{+}(L)=\left\{x \in L \mid x^{+}=1\right\}$ and it is an ideal of $L$. The core of $L$ is $C(L)=\left\{x \in L \mid x^{+}=1, x^{*}=0\right\}$ and $\operatorname{Cen}(L)$ is the Boolean lattice of the complemented elements of $L$. If $a \in L$ the elements $a^{n(+*)}$, with $n \in N_{0}$, are defined in $L$, inductively, as follows: $a^{0(+*)}=a$ and $a^{(n+1)(+*)}=\left(a^{n(+*)}\right)^{+*}$. We say that $L$ has finite range if, for every $a \in L$, there is $k \in N_{0}$ such that $a^{(k+1)(+*)}=a^{k(+*)}$, this is equivalent to $a^{k(+*)} \in \operatorname{Cen}(L)$.

Observation. Let $L \in \mathbf{B}_{\omega}^{\omega}$ and $X$ its dual space.

(i) Let $U \in \mathcal{O}(X)$. Identifying $L$ and $\mathcal{O}(X)$ we have that

$U \in D^{+}(L)$ if and only if $U \subseteq X-\operatorname{Max} X$;

$U \in C(L)$ if and only if $\operatorname{Min} X \subseteq U \subseteq X-\operatorname{Max} X$;

$U \in \operatorname{Cen}(L)$ if and only if $U$ is an up set;

for all $n \in N_{0}, U^{n(+*)}=X-(\uparrow \downarrow)^{n}(X-U)$.

Therefore

$D^{+}(L)$ is principal if and only if $X-\operatorname{Max} X$ is closed (therefore clopen);

$\mathrm{C}(\mathrm{L}) \neq \emptyset$ if and only if $\operatorname{Min} X \subseteq X-\operatorname{Max} X$;

$L$ has finite range if and only if, for every clopen up set $V$ of $X$, there exists $k \in N_{0}$ such that $(\uparrow \downarrow)^{k+1}(V)=(\uparrow \downarrow)^{k}(V)$, that is $(\uparrow \downarrow)^{k}(V) \in \operatorname{Cen}(L)$.

(ii) The open up-down subsets of $X$ are the sets of the form $\bigcup_{n \in N_{0}}(\uparrow \downarrow)^{n}(A)$ where $A$ is a clopen up set if and only if every normal filter $F$ of $L$ is principal (i.e., there is $a \in L$ such that $\left.F=\left\{x \in L \mid\left(\exists n \in N_{0}\right) x \geqq a^{n(+*)}\right\}\right)$.

(iii) $l(X) \leqq 3$ if and only if the poset of all prime ideals of $L$ contains no 5-element chain. 
Proposition 3.11. Let $L \in \mathrm{B}_{\omega}^{\infty}$ and $X$ be its dual space. If $\operatorname{Mid} X$ is finite, then every determination class of $L$ is finite.

Proof. Suppose that Mid $X$ is finite. We identify $L$ and $\mathcal{O}(X)$. Now, let $U_{0} \in \mathcal{O}(X)$. A clopen down set $U$ is in the determination class of $U_{0}$ if and only if $U-\operatorname{Mid} X=$ $U_{0}-\operatorname{Mid} X$. Hence, since $\operatorname{Mid} X$ is finite, there is a finite number of elements in the determination class of $U_{0}$.

Proposition 3.12. Let $X$ be the dual space of $L \in \mathbf{B}_{\omega}^{\omega}$, Suppose that there is an open set $V$ satisfying the following conditions

(i) $\operatorname{Min} X \cap \operatorname{Max} X \subseteq V$;

(ii) $V$ is contained in every clopen down set $U$ such that $\operatorname{Min} X \subseteq U$.

If every determination class of $L$ is finite, then $\mathrm{Mid} X$ is finite.

Proof. Let us identify $L$ and $\mathcal{O}(X)$. Suppose that $\operatorname{Mid} X$ is infinite. Either $\operatorname{Mid} X$ does not satisfy, at least, one of the chain conditions or Mid $X$ has an infinite antichain. First, suppose that Mid $X$ does not satisfy the descending chain condition, i.e., there are $y_{n} \in \operatorname{Mid} X$, with $n \in N$, such that $y_{n+1}<y_{n}$. For each $n \in N$, consider

$$
\Gamma_{n}=\operatorname{Min} X \cup\left\{y_{n+1}\right\} \text { and } \Delta_{n}=(\operatorname{Max} X \cap(X-V)) \cup\left\{y_{1}, \ldots, y_{n}\right\},
$$

which are closed subsets of $X$. For all $x \in \Gamma_{n}$ and $z \in \Delta_{n}$, we have $z$. The total order disconnectedness and compactness provides, for each $n \in N$, a clopen down set $U_{n}$ such that $\Gamma_{n} \subseteq U_{n} \subseteq X-\Delta_{n}$. Now let $m, n$ be distinct natural numbers. It is obvious that $U_{n} \neq U_{m}$. Next notice that if $x \in \operatorname{Min} X$, then $x \in U_{n}$ and $x \in U_{m}$. Also, if $x \in \operatorname{Max} X-$ $\operatorname{Min} X$, then, by [5, Lemma 10.16], there is a clopen down set $U$ such that $\operatorname{Min} X \subseteq U$ and $x \notin U$. Hence, by (2), we have that $x \notin V$ and then $x \notin U_{n} \cup U_{m}$. Thus, $U_{n}-\operatorname{Mid} X=$ $U_{m}-\operatorname{Mid} X$. Therefore, the clopen down sets $U_{n}$, with $n \in N$, are in the same determination class, a contradiction.

Second, let us assume that $\mathrm{Mid} X$ does not satisfy the ascending chain condition or that $\operatorname{Mid} X$ has an infinite antichain. Then there are $y_{n} \in \operatorname{Mid} X$, with $n \in N$, such that $y_{n+1} y_{n}$. For each $n \in N$, consider $\Gamma_{n}=\operatorname{Min} X \cup\left\{y_{1}, \ldots, y_{n}\right\}$ and $\Delta_{n}=(\operatorname{Max} X$ $\cap(X-V)) \cup\left\{y_{n+1}\right\}$. Reasoning as before leads us once again to a contradiction.

At this point we observe that if $L$ is such that $C(L) \neq \emptyset$, then we may consider $V=\emptyset$ and so it is obvious that the sets $U_{n}, n \in N$, of last proof are in $C(L)$. Thus we have the following

Corollary 3.13. Let $L \in \mathbf{B}_{\omega}^{\infty}$ be such that $C(L) \neq \emptyset$ and let $X$ be its dual space. If $C(L)$ is finite, then Mid $X$ is finite.

Corollary 3.14. Let $L \in \mathrm{B}_{\omega}^{\infty}$ be such that $D^{+}(L)$ is principal and let $X$ be its dual space. If every determination class of $L$ is finite, then $\mathrm{Mid} X$ is finite. 
Proof. If $D^{+}(L)$ is principal, then $X-\operatorname{Max} X$ is clopen and so is $\uparrow((X-\operatorname{Max} X)$ $\cap \operatorname{Min} X)$. As $\uparrow((X-\operatorname{Max} X) \cap \operatorname{Min} X)=X-(\operatorname{Min} X \cap \operatorname{Max} X)$, we have that $\operatorname{Min} X$ $\cap \operatorname{Max} X$ is clopen and so it is open. The result now follows from Proposition 3.12, by taking $V=\operatorname{Min} X \cap \operatorname{Max} X$.

Corollary 3.15. Let $L \in \mathbf{B}_{\omega}^{\omega}$ be such that $L$ has finite range and $\operatorname{Cen}(L)$ is finite and let $X$ be the dual space of $L$. Then if every determination class of $L$ is finite so is Mid $X$.

Proof. We identify $L$ and $\mathcal{O}(X)$. Let $I=\{U \in \mathcal{O}(X) \mid \operatorname{Min} X \subseteq U\}$. For every $U \in I$, there is $k(U) \in N_{0}$ such that $U^{k(U)(+*)} \in \operatorname{Cen}(L)$. Consider $\mathscr{A}=\left\{U^{k(U)(+*)} \mid U \in I\right\}$ and $V=\cap \mathscr{A}$. Since $\operatorname{Cen}(L)$ is finite, so is $\mathscr{A}$. Thus $V$ is an open subset of $X$. If $U \in I$, then $V=\cap \mathscr{A} \subseteq U^{k(U)(+*)} \subseteq U$ and

$$
U^{k(U)(+\bullet)}=X-(\uparrow \downarrow)^{k(U)}(X-U) \supseteq X-(\uparrow \downarrow)^{k(U)}(X-\operatorname{Min} X) \supseteq \operatorname{Min} X \cap \operatorname{Max} X .
$$

Therefore, $V=\cap \mathscr{A} \supseteq \operatorname{Min} X \cap \operatorname{Max} X$ and, by Proposition 3.12, the set Mid $X$ is finite.

Proposition 3.16. Let $L \in \mathbf{B}_{\omega}^{\omega}$ be such that $L$ has finite range. Then the following conditions are equivalent

(i) every normal filter of $L$ is principal;

(ii) every open up-down subset of the dual space of $L$ is closed;

(iii) $\operatorname{Cen}(L)$ is finite.

Proof. Let $X$ be the dual space of $L$ and identify $L$ with $\mathcal{O}(X)$.

(i) $\Rightarrow$ (ii) Let $\Gamma$ be an open up-down subset of $X$. Then, by Observation (ii), there is a clopen up set $A$ such that $\Gamma=\bigcup_{n \in N_{0}}(\uparrow \downarrow)^{n}(A)$. Since $\Gamma$ has finite range, we have $\Gamma=(\uparrow \downarrow)^{k}(A)$, for some $k \in N_{0}$. Thus $\Gamma$ is closed in $X$.

(ii) $\Rightarrow$ (i) This is obvious, by Observation (ii).

(ii) $\Rightarrow$ (iii) We prove that the Boolean lattice $\operatorname{Cen}(L)$ satisfies the ascending chain condition and, that, consequently, $\operatorname{Cen}(L)$ is finite. Let $U_{n}, n \in N$, be clopen up-down subsets of $X$ such that $U_{n} \subseteq U_{n+1}$. Consider the open up-down set $\Gamma=\bigcup_{n \in N} U_{n}$. The set $\Gamma$ must be closed. Now, since $X$ is compact and $\left\{U_{n} \mid n \in N\right\}$ is a chain, we have $\Gamma=U_{s}$, for some $s \in N$. Therefore, $\operatorname{Cen}(L)$ satisfies the ascending chain condition.

(iii) $\Rightarrow$ (ii) Let $\Gamma$ be an open up-down subset of $X$ and $I=\{U \in \mathcal{O}(X) \mid X-U \subseteq \Gamma\}$. For each $U \in I$, there is $k(U) \in N$ such that $(\uparrow \downarrow)^{k(U)}(X-U) \in \operatorname{Cen}(L)$. Let $V_{U}=(\uparrow \downarrow)^{k(U)}(X-U)$. The centre of $L$ contains $\left\{V_{U} \mid U \in I\right\}$, which must be finite. Consider $A=\bigcup_{U \in I} V_{U}$. This is a clopen up-down set contained in $\Gamma$. Now let $x \in \Gamma$. The set $X-\Gamma$ is a closed down set and $x \notin X-\Gamma$. Then, by [5, Lemma 10.16], there is a clopen down set $U$ such that $X-\Gamma \subseteq U$ and $x \notin U$, i.e., $x \in X-U$ and $U \in I$. Thus $x \in A$ and $\Gamma=A$. Therefore, $\Gamma$ is closed in $X$, as required. 


\section{PRINCIPAL CONGRUENCES ON DISTRIBUTIVE DOUBLE p-ALGEBRAS 501}

Corollary 3.17. [2, Theorem 4.3] Let $L \in B_{\omega}^{\omega}$ be such that $D^{+}(L)$ is a principal ideal. Then $L$ has $P C$ if and only if

(i) every normal filter of $L$ is principal;

(ii) every determination class of $L$ is finite;

(iii) there is no 5-element chain in the poset of prime ideals of $L$.

Proof. This is an immediate consequence of Theorem 3.9, Observation (ii) and (iii), Proposition 3.11 and Corollary 3.14 .

In [2, Corollary 4.4], Beazer characterizes the distributive double $p$-algebras with finite range and $D^{+}(L)$ principal that have $P C$. In the next corollary, we show that requiring $D^{+}(L)$ principal is not necessary. In fact we are able to describe, in the same algebraical way, all the distributive double $p$-algebras with the finite range that have $P C$. if

Corollary 3.18. Let $L \in \mathbf{B}_{\omega}^{\omega}$ be such that $L$ has finite range. Then $L$ has $P C$ if and only

(i) $\operatorname{Cen}(L)$ and every determination class are finite;

(ii) there is no 5-element chain in the poset of all prime ideals of $L$.

Proof. It follows from Theorem 3.9, Observation (ii) and (iii), Propositions 3.11 and 3.16 and Corollary 3.15 .

Corollary 3.19. Let $L \in \mathbf{B}_{\omega}^{\omega}$ be such that its p-algebra reduct is Stone. Then $L$ has $P C$ if and only if conditions (i) and (ii) in the statement of Corollary 3.18 hold.

Proof. We start by noticing that if the p-algebra reduct of $L$ is Stone, then $L$ has finite range and then we apply Corollary 3.18 .

In [2, Corollary 4.5] not only conditions (i) and (ii) are required but also is the existence of an element $t \in D^{+}(L)$ such that $D^{+}(L) \subseteq\left(t^{* *}\right]$. However, we observe that if $L$ is a distributive double $p$-algebra whose $p$-algebra reduct is Stone and $\operatorname{Cen}(L)$ is finite, there is always an element $t \in D^{+}(L)$ such that $D^{+}(L) \subseteq\left(t^{* *}\right]$. In fact, if we identify $L$ and $\mathcal{O}(X)$, then $I=\left\{U^{* *} \mid U \in D^{+}(L)\right\}$ is contained in $\operatorname{Cen}(L)$ and so $I$ is finite. Next, for each $\Gamma \in I$ we choose $U_{\Gamma} \in D^{+}(L)$ such that $U_{\Gamma}^{* *}=\Gamma$. Hence $V=\bigcup_{\Gamma \in I} U_{\Gamma} \in D^{+}(L)$ and, for every $U \in D^{+}(L)$, we have that $U \subseteq U^{* *} \subseteq V^{* *}$. Therefore $D^{+}(L) \subseteq\left(V^{* *}\right]$, as required.

In [3], Blyth and Varlet showed that a distributive lattice $L$ has $P C$ if and only if $L$ is finite and $l(J(L)) \leqq 1$. Therefore the next corollary corresponds to Theorem 4.9 of [2].

Corollary 3.20. [2, Theorem 4.9] Let $L \in \mathbf{B}_{\omega}^{\omega}$ be such that $C(L)=\emptyset$. Then $L$ has $P C$ if and only if

(i) every normal filter of $L$ is principal;

(ii) $C(L)$ is finite and $l(J(C(L))) \leqq 1$. 
Proof. First we prove that $\operatorname{Mid} X$ is finite and $l(X) \leqq 3$ if and only if $C(L)$ is finite and $l(J(C(L))) \leqq 1$. Let us assume that $\mathrm{Mid} X$ is finite and $l(X) \leqq 3$. By Proposition 3.11, we have that $C(L)$ is finite as $C(L)$ is a determination class. Suppose that $l(J(C(L)))>1$. Let $U_{n}$, with $1 \leqq n \leqq 3$, be elements of $J(C(L))$ such that $U_{1} \subset U_{2} \subset U_{3}$. For $n=2,3$, consider the closed down set $V_{n}=\downarrow\left(U_{n}-U_{n-1}\right) \cup \operatorname{Min} X$. Each $V_{n}$ is open, since $V_{n}=X-\left(\operatorname{Max} X \cup Y_{n}\right)$ for some $Y_{n} \subseteq \operatorname{Mid} X$ and $\operatorname{Mid} X$ is finite and so is $Y_{n}$. Thus $V_{n} \in C(L)$. Obviously, $U_{n}=V_{n} \cup U_{n-1}$, for $n=2,3$. As $U_{n} \in J(C(L))$, for $1 \leqq n \leqq 3$, we have

$$
\operatorname{Min} X \subset U_{1}, U_{3} \subseteq X-\operatorname{Max} X, U_{2}=V_{2} \text { and } U_{3}=V_{3} \text {. }
$$

Next let $x_{1} \in U_{1}-\operatorname{Min} X$. Since $U_{1} \subset U_{2}=V_{2}=\left(\downarrow\left(U_{2}-U_{1}\right) \cup \operatorname{Min} X\right)$, there is $x_{2} \in U_{2}-$ $U_{1}$ such that $x_{1}<x_{2}$. Similarly, there is $x_{3} \in U_{3}-U_{2}$ such that $x_{2}<x_{3}$. On the other hand, every element of $X$ contains a minimal one and it is contained in a maximal one. Therefore, $l(X) \geqq 4$, which is a contradiction.

Conversely, suppose that $C(L)$ is finite and $l(J(C(L))) \leqq 1$. By Corollary 3.13 , it follows that Mid $X$ is finite. Suppose that $l(X)>3$. Let $x_{n}$, with $1 \leqq n \leqq 5$, be elements of $X$ such that $x_{1}<x_{2}<x_{3}<x_{4}<x_{5}$. For $n \in\{2,3,4\}$, consider the closed down set $U_{n}=\left(\downarrow x_{n}\right) \cup$ $\operatorname{Min} X$. Now each $U_{n}$ is open, since $U_{n}=X-\left(\operatorname{Max} X \cup Y_{n}\right)$ for some $Y_{n} \subseteq \operatorname{Mid} X$. Thus, it is obvious that $U_{n} \in J(C(L))$, for $n \in\{2,3,4\}$, and $U_{2} \subset U_{3} \subset U_{4}$, a contradiction.

Finally, the result follows from Theorem 3.9 and Observation (ii).

Corollary 3.21. [2, Corollary 4.10] Let $L \in B_{\omega}^{\omega}$ be such that $L$ has finite range and $C(L) \neq \emptyset$. Then $L$ has $P C$ if and only if

(i) $\operatorname{Cen}(L)$ is finite;

(ii) $C(L)$ is finite and $l(J(C(L))) \leqq 1$.

Proof. This is an immediate consequence of Corollary 3.20 and Proposition 3.16.

\section{REFERENCES}

1. M. E. Adams, Principal congruences in de Morgan algebras, Proc. Edinburgh Math. Soc. 30 (1987), 415-421.

2. R. BEAzER, Some p-algebras and double p-algebras having only principal congruences, Glasgow Math. J. 34 (1992), 157-164.

3. T. S. Blyth and J. C. Varlet, Principal congruences on some lattice-ordered algebras, Discrete Math. 81 (1990), 323-329.

4. B. A. DaveY, Subdirectly irreducible distributive double p-algebras, Algebra Universalis 8 (1978), 73-88.

5. B. A. Davey and H. A. Priestley, Introduction to Lattices and Order (Cambridge University Press, Cambridge, 1990).

6. M. S. Goldberg, Distributive p-algebras and Ockham algebras: a Topological Approach (Ph.D. thesis, La Trobe University, Bundoora, Australia, 1979).

7. H. A. Priestley, Stone lattices: a topological approach, Fund. Math. 84 (1974), 127-143. 
PRINCIPAL CONGRUENCES ON DISTRIBUTIVE DOUBLE p-ALGEBRAS 503

8. H. A. Priestley, The construction of spaces dual to pseudocomplemented distributive lattices, Quart. J. Oxford 26 (1975), 215-228.

9. J. VAZ de Carvalho, Congruências principais em álgebras de Stone duplas, Actas $X V$ Jornadas Luso-Espanholas de Matemática 1 (1990), 49-54.

Centro de Álgebra da Universidade de Lisboa

Av. Prof. Gama Pinto, 2

1699 LisBoa CODEX

Portugal 